\title{
Shape optimization of photoacoustic resonators
}

\author{
B. Kost, B. Baumann, M. Germer \& M. Wolff \\ Department of Mechanical and Production Engineering, \\ Hamburg University of Applied Sciences, Germany
}

\begin{abstract}
The sensitivity of photoacoustic sensors strongly depends on the shape of the acoustical resonator. Up to now, mainly photoacoustic sensors consisting of a number of cylindrical parts have been investigated (cylinder cells, $\mathrm{H}$ cells, $\mathrm{T}$ cells etc.). In this paper, a numerical shape optimization of the resonator cell of photoacoustic sensors is described. The approach considers all shapes that can be represented by a number of axisymmetrical truncated cones which are connected in a continuous way. In addition, the geometry of the cell is subjected to certain constraints, e.g. the laser beam should not be blocked during its passage through the cell. The purpose is to maximize the sensor's signal strength. The acoustic pressure at the microphone of the sensor represents the objective function and is calculated using an eigenmode expansion combined with a finite element calculation. The solution of the 9-dimensional nonlinear optimization problem is a resonator shape with a substantial quality improvement with reference to the well-known $\mathrm{H}$ cell. Keywords: photoacoustic, gas sensor, acoustic resonator, shape optimization.
\end{abstract}

\section{Introduction}

The photoacoustic effect, described by Bell as early as 1880 [1], can be used to develop sensors for trace gas analysis. There is a broad field of application for photoacoustic sensors, e.g. medical breath tests and environmental pollution control.

The general functional principle of a photoacoustic sensor is simple and is outlined in Figure 1. The sensor consists mainly of four parts: a photoacoustic cell, a microphone, a light source (laser) and a chopper. The cell contains a sample of the gas under investigation. The laser beam modulated by the chopper passes through a transparent window in the cell's front side. The laser wavelength is adjusted to an 


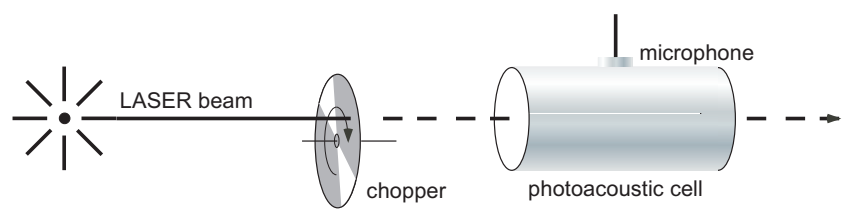

Figure 1: Design of photoacoustic sensor with cylindrical resonator cell.

absorption line of the trace gas causing molecular excitations accompanied by heat production. The periodical heat variation due to the chopper excites sound waves in the cell. The pressure field of the sound waves can be detected by the microphone and is equivalent to the signal strength of the sensor. The chopper frequency and the frequency of the wave propagation are identical. In order to amplify the microphone signal it is evident to adjust the chopper frequency to an acoustic resonance of the measuring cell, hence the name photoacoustic resonator. Details of the photoacoustic technique can be found in [2].

So far, mainly photoacoustic cells consisting of a number of cylindrical parts have been investigated. The most prominent example is a photoacoustic sensor with a cylindrical resonator cell. Another sensor design combines a cylindrical resonator with two cylindrical buffer volumes at the ends, known as $\mathrm{H}$ cell [3]. It is described in detail in section 3. Recently, the interest in photoacoustic sensors with unconventional design is increasing. The so-called $\mathrm{T}$ cell $[4,5]$ consists of two perpendicular arranged cylinders, one serving as the absorption cell, the other as resonator cell. A variation of the $\mathrm{T}$ cell is the $\mathrm{X}$ cell where the resonator cell has been split-up in two parts [6].

In order to further enhance the signal strength and sensitivity of a photoacoustic sensor in the following a numerical shape optimization of the resonator cell has been carried out. For that purpose the resonator cell is modeled by a number of cones. Details of the approach can be found in section 4.

\section{Analysis of photoacoustic resonators}

\subsection{Calculation of the signal strength}

Simple cylinder shaped resonators can be treated analytically. For cells of more complex shape, an analytical examination is impossible. Therefore, the investigation has to rely on numerical methods. Recently, a complete numerical description of the photoacoustic signal generation process including the computation of signal strength has been achieved $[5,7,8]$. In the following the numerical analysis of this approach is summarized.

In a first step the acoustical eigenmodes of the resonator cell have to be computed. The pressure field of the photoacoustical cell can be expressed by a weighted superposition of the acoustical eigenmodes. This leads to the amplitude resonance function of the resonator. According to experience, consideration of the first eight 
Table 1: Gas parameters of $\mathrm{N}_{2}$ under atmospheric conditions $(T=293 \mathrm{~K}, p=$ $1013 \mathrm{hPa}$ ) [11].

\begin{tabular}{ll}
\hline \hline density $\rho$ & $1.15 \mathrm{~kg} / \mathrm{m}^{3}$ \\
sound velocity $c$ & $343 \mathrm{~m} / \mathrm{s}$ \\
viscosity $\eta$ & $1.76 \cdot 10^{-5} \mathrm{Pas}$ \\
coefficient of heat conduction $\kappa$ & $2.56 \cdot 10^{-2} \mathrm{~W} / \mathrm{m} \mathrm{K}$ \\
specific heat capacity at constant volume $c_{\mathrm{V}}$ & $0.741 \cdot 10^{3} \mathrm{~J} / \mathrm{kg} \mathrm{K}$ \\
specific heat capacity at constant pressure $c_{\mathrm{p}}$ & $1.038 \cdot 10^{3} \mathrm{~J} / \mathrm{kg} \mathrm{K}$ \\
\hline \hline
\end{tabular}

eigenmodes has been found to be sufficient. The maximum value of the amplitude resonance function at a nontrivial resonance frequency can be considered to be the signal strength of the photoacoustical resonator cell.

For the evaluation of the amplitude resonance function the inclusion of loss is necessary. This has been done by means of a quality factor approach [9]. StokesKirchhoff loss due to viscosity and thermal conduction as well as the thermal conductivity surface loss and the surface loss due to viscosity have been considered in this way.

The numerical calculations were carried out using the finite element tool COMSOL Multiphysics 3.4 [10]. The results were obtained with the default solver parameters.

\subsection{Basic data}

In the following simulations it is assumed that the cells are filled with nitrogen $\mathrm{N}_{2}$ with traces of $\mathrm{NO}$ under atmospheric conditions. The gas parameters of $\mathrm{N}_{2}$ are taken from [11] and can be found in Table 1.

To obtain proper laser data an experimental investigation has been carried out. The investigation of our set-up reveals that the cross-sections of the laser beam intensity are nearly circularly Gaussian profiles. The effective radius has been found to be approximately $1.7 \mathrm{~mm}$ independent of the axial position.

The intensity decrease in the longitudinal direction is given by an exponential function using a decay factor $\alpha$. The absorption coefficient of NO which is responsible for the decrease of the laser intensity along the cell axis is $\alpha=0.166 \mathrm{~m}^{-1}$ [12]. This quantity represents also a measure of the NO concentration in the cell.

\section{Reference cell}

For the numerical evaluation of the solution quality of the shape optimization described below the well-known $\mathrm{H}$ cell [3] has been taken as a reference. The small-sized cylinder of diameter $d_{1}$ in the middle represents the resonator. At both ends are larger cylinders of diameter $d_{2}$ serving as buffer volumes. A sectional 

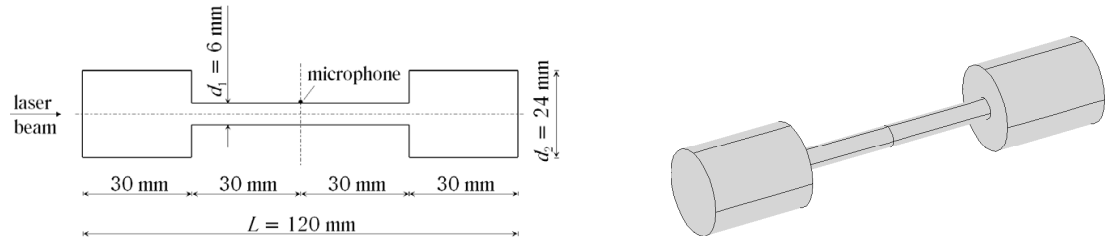

Figure 2: Reference cell: design data (left), 3D model (right).

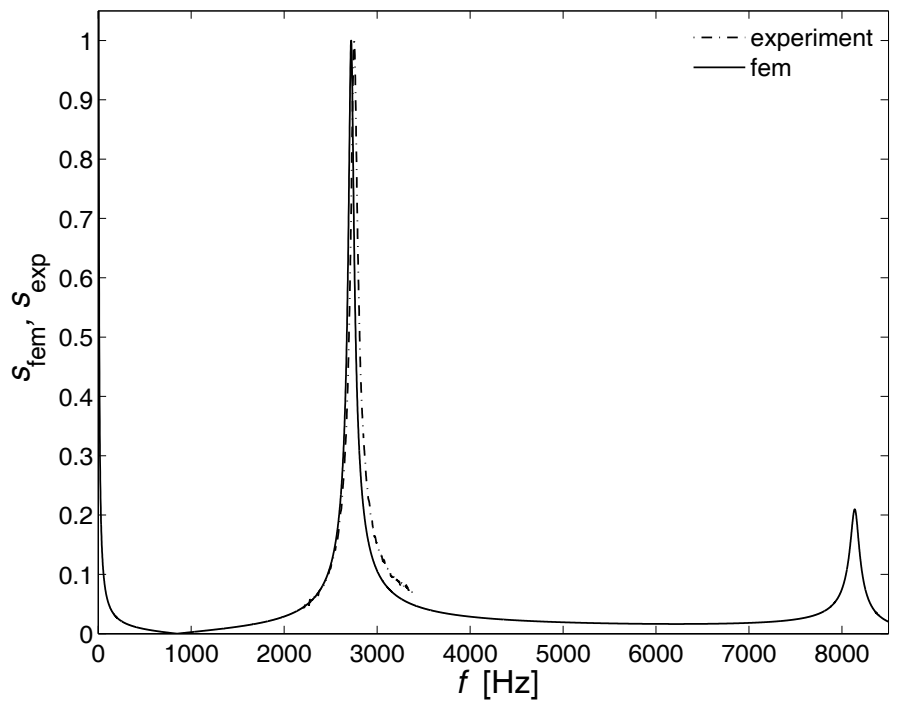

Figure 3: Comparison of normalized amplitude resonance function $s_{\mathrm{fem}}(f)$ and microphone signal function $s_{\exp }(f)$ for the reference cell over the numerically analyzed frequency range.

drawing of the reference cell with the design data used in this investigation is shown in Figure 2 (left). A 3D model can be found in Figure 2 (right).

The quality of the reference cell has been measured experimentally in our laboratory over a certain range of the chopper frequency $f$. Additionally, the cell has been analyzed by the finite element eigenmode expansion approach described in section 2 over a larger range of $f$. This has been done to cover a sufficient number of eigenmodes in the series expansion. Both results, the experimentally obtained microphone signal function $s_{\exp }(f)$ and the numerically calculated amplitude resonance function $s_{\text {fem }}(f)$, are depicted in Figure 3. To allow a reasonable comparison the data have been normalized with respect to the maximum value of each function.

The numerical and experimental data are in good accordance. The frequency of the experimental signal maximum is $2753 \mathrm{~Hz}$, the frequency of the numerical 

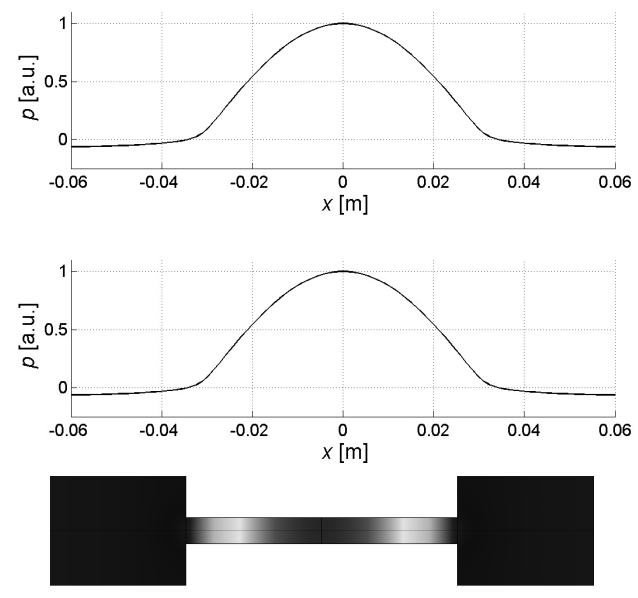

Figure 4: Dominant eigenmode of the reference cell $\left(2^{\text {nd }}\right.$ nontrivial mode at $2721 \mathrm{~Hz}$ ), acoustic pressure distribution $p$ along cell axis $x$ (top), mode shape $|p|$ (bottom).

resonance maximum is $2721 \mathrm{~Hz}$. This is a relative deviation of approximately $1.2 \%$. The graphs of the functions are quite similar. The width at the peak of the experimental function is slightly larger than that of the numerical function. Since the width of the function is determined by loss effects and the finite element simulation does not include all effects that contribute to loss of the photoacoustic signal, this is an expected characteristic. However, the good accordance of the numerical and experimental data shows that the most important loss effects have been considered in the finite element model. Further investigations concerning the comparison of analytical, experimental and finite element results of certain photoacoustic cells can be found in $[5,7]$.

In Figure 4 (bottom) the dominant eigenmode of the amplitude resonance function is shown, the $2^{\text {nd }}$ nontrivial mode. It consists mainly of a longitudinal mode of the center cylinder. The according eigenfrequency of a cylinder with both ends open and a length of $60 \mathrm{~mm}$ can be analytically determined to $2858 \mathrm{~Hz}$. Figure 4 (top) shows the acoustic pressure distribution along the cell axis. The distance $>60 \mathrm{~mm}$ between the zero crossings of the function is equivalent to an effective length of the middle cylinder, hence the lower frequency $2721 \mathrm{~Hz}$. More about end corrections can be found in [13].

\section{Shape optimization model}

The finite element model for shape optimization of a photoacoustic cell consists of 8 axisymmetrical truncated cones of equal length $\frac{L}{8}$. The cones are connected in a continuous way. The 9 radii $x_{i}(i=1,2, \ldots, 9)$ of the cones represent the optimization variables. In Figure 5 a $3 \mathrm{D}$ model of a potential resonator cell is shown. 


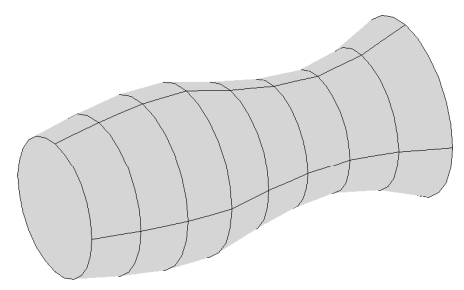

Figure 5: 3D model of a potential resonator cell contained in the search space.

The objective function

$$
z: \mathbb{R}^{9} \longrightarrow \mathbb{R}, \quad \boldsymbol{x} \longrightarrow z(\boldsymbol{x})=: Z
$$

of the shape optimization problem is set to $z:=\left|p_{\mathrm{m}}\right|$. The function $p_{\mathrm{m}}$ determines the value of the pressure field at the microphone position. This is a measure of the signal strength of the photoacoustic cell. According to this, the objective function value $Z$ can be calculated by the procedure described in section 2.1 .

The optimization parameters have to fulfill the inequaltities

$$
r_{\mathrm{lb}} \leq x_{i}, \quad i=1,2, \ldots, 9
$$

where $r_{\mathrm{lb}}=3 \mathrm{~mm}$ is a lower bound for the radii. The value $r_{\mathrm{lb}}$ is sufficiently larger than the laser beam radius. These constraints have been imposed to prevent that the laser beam is blocked during its passage through the cell and to ensure a proper energy input into the system. Due to technical reasons the radii have also been restricted by upper bounds

$$
x_{i} \leq r_{\mathrm{ub}}, \quad i=1,2, \ldots, 9
$$

with $r_{\mathrm{ub}}=60 \mathrm{~mm}$. This guarantees a compact design of the photoacoustic cell.

With the upper and lower bounds the subset $\mathbf{X} \subset \mathbb{R}^{9}$ defined by

$$
\mathbf{X}:=\left\{\boldsymbol{x} \in \mathbb{R}^{9} \mid r_{\mathrm{lb}} \leq x_{i} \leq r_{\mathrm{ub}}, i=1,2, \ldots, 9\right\}
$$

is the feasible region. A solution of the nonlinear maximization problem can be expressed by

$$
\boldsymbol{x}^{*}:=\operatorname{argmax}\{z(\boldsymbol{x}) \mid \boldsymbol{x} \in \mathbf{X}\} \quad \text { and } \quad Z^{*}:=z\left(\boldsymbol{x}^{*}\right) .
$$

\section{Optimization results}

The shape optimization problem of section 4 has been solved with the MATLAB [14] standard optimization function for constrained problems fmincon. Preliminary investigations show that the shape optimization problem of photoacoustic resonator cells may have multiple local optima [15]. Therefore, the optimization has been rerun with different initial shapes, representing different starting points 
in the search space. For all initial shapes the smallest radii have been set to $3 \mathrm{~mm}$, the largest to $12 \mathrm{~mm}$.

It seems obvious to start the optimization at a cylindrical resonator shape. However, this is problematic because due to the axial symmetry combined with an approximate translation symmetry of the laser intensity distribution in a cylindrical resonator only radial modes can be excited. The low value of the absorption coefficient and the small length of the cell causes only a marginal decrease of the laser intensity of about $2 \%$. Therefore, an effective excitation of longitudinal or azimuthal modes is not possible. In practice impurities in the gas, deviations from the symmetry due to the laser adjustment and other factors lead to a weak excitation of nonradial modes. However, the unsatisfying performance of the cylinder cell can be explained by the facts mentioned above. The eigenfrequencies of the radial modes are very high and with respect to the numerical investigations of the photoacoustic sensor beyond the interesting frequency range.

A collection of initial shapes used in this investigation and resulting solutions are depicted in Table 2 together with the ratio

$$
\zeta:=\frac{Z^{*}}{Z_{\mathrm{ref}}} .
$$

The parameter $\zeta$ specifies the solution quality in comparison to the reference cell. $Z_{\text {ref }}$ is the maximum value of the not normalized amplitude resonance function of the $\mathrm{H}$ cell resonator.

Even though the decrease of laser intensity along the cell axis is weak, it could not be excluded that it effects the appearance and quality of the solution. Therefore, albeit similar, both geometries (cases 1 and 2 of the table) have been chosen as initial shapes. However, the obtained solutions are similar too and of poor quality. Case 3 has been included in the collection of initial shapes with the intention to have a starting point which is in the limits of our modeling close to the reference cell. The result shows a decent improvement of the signal strength of about $20 \%$. Case 4 and 5 have been chosen to provoke the exploration of different parts of the search space. Although, the solution geometry of case 4 looks rather different compared to case 3 the $\zeta$-value is only slightly better. The best solution quality exhibits case 5 . Compared to the reference cell an improvement of about $31.6 \%$ could be found. In Figure 6 a 3D model of the solution is depicted. The best geometry found is an asymmetrical shape with horn-like ends.

In Figure 7 (bottom) the dominant eigenmode of the amplitude resonance function of the best shape is shown, the $4^{\text {th }}$ nontrivial mode. Figure 7 (top) shows the acoustic pressure distribution along the cell axis.

\section{Conclusions}

In the present article we described the numerical shape optimization of the resonator cell of axisymmetrical photoacoustic sensors. The shape optimization problem of such sensor cells is nonconvex, hence multiple local optima exist. To ensure 
Table 2: Longitudinal sections of initial shapes and solutions. $\zeta$ is the solution quality ratio, see Eq. (6).

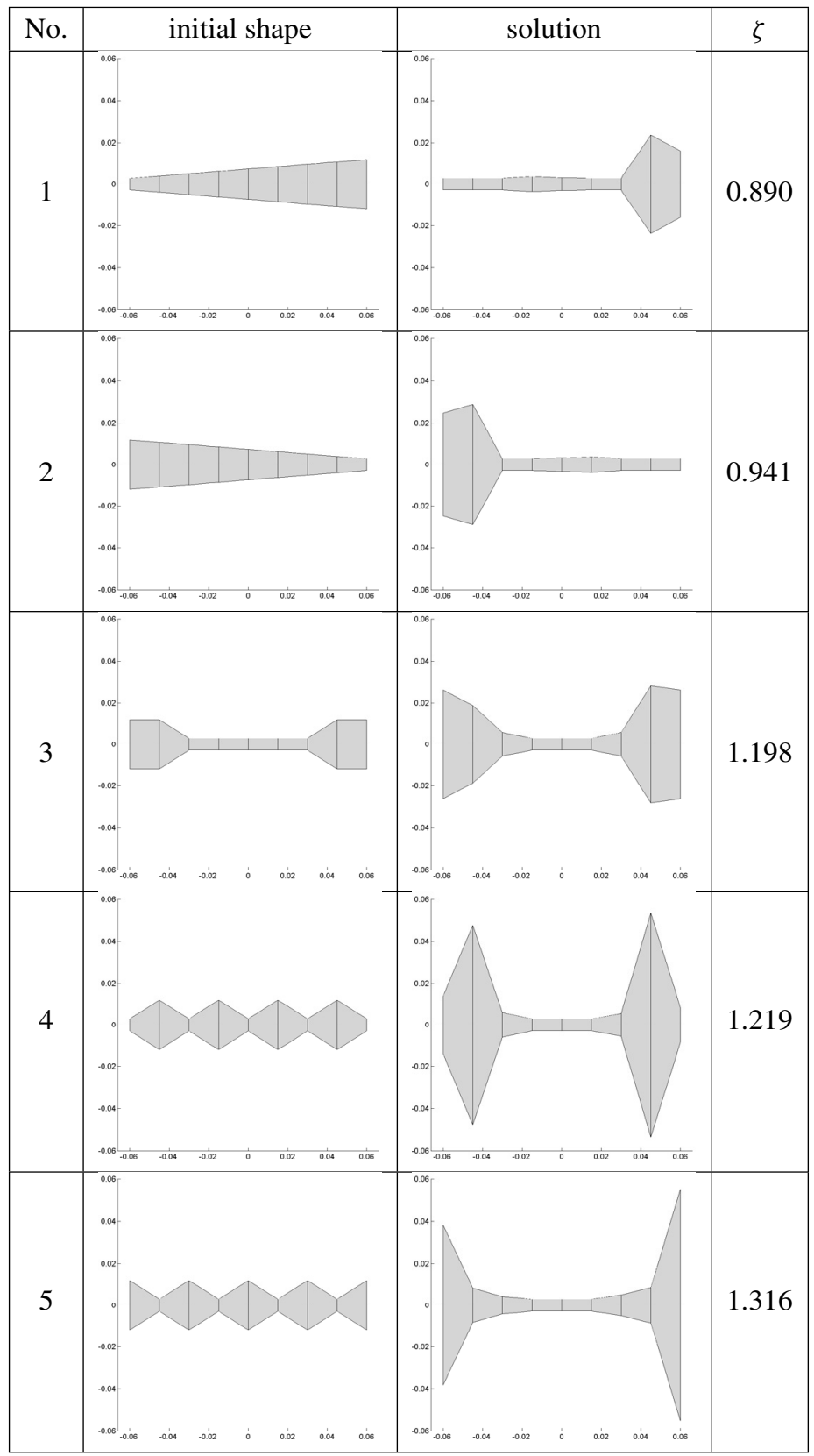




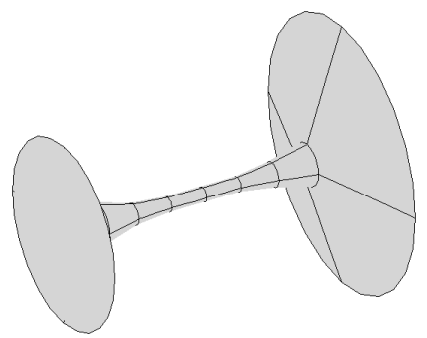

Figure 6: 3D model of best resonator shape.
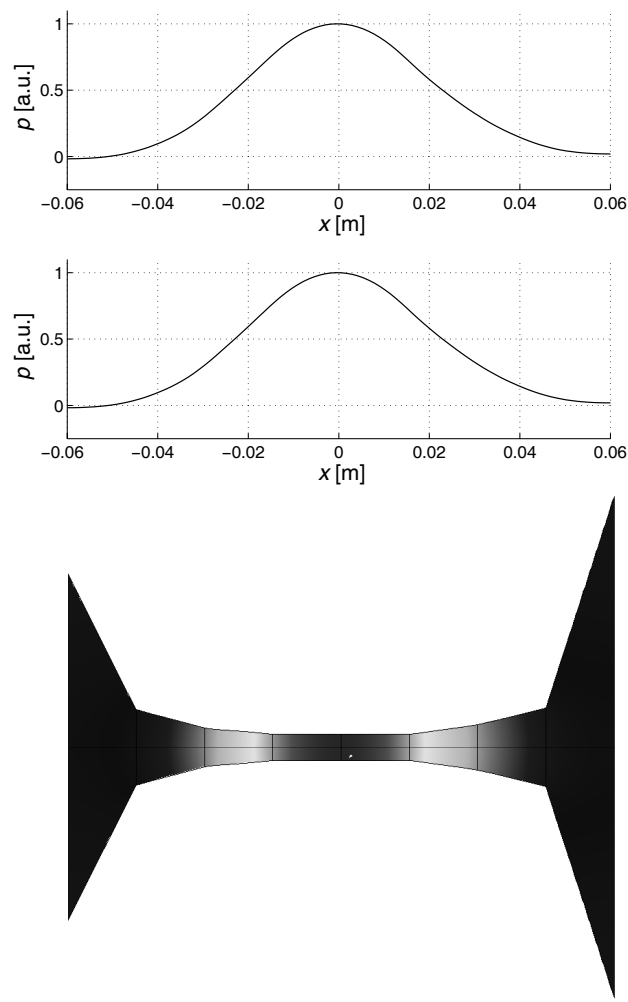

Figure 7: Dominant eigenmode of best cell shape ( $4^{\text {th }}$ nontrivial mode at $2673 \mathrm{~Hz}$ ), acoustic pressure distribution $p$ along cell axis $x$ (top), mode shape $|p|$ (bottom).

an adequate exploration of the search space a collection of initial shapes have been used. The best solution found is a resonator shape with horn-like ends. With respect to the well-known $\mathrm{H}$ cell this cell shows a considerably improvement in signal strength of about $32 \%$. An experimental verification of this result is planned. 


\section{References}

[1] Bell, A.G., On the production and reproduction of sound by light. Am. J. Science, 20, pp. 305-309, 1880.

[2] Michaelian, K.H., Photoacoustic Infrared Spectroscopy. Wiley-Interscience: Hoboken, 2003.

[3] Nodov, E., Optimization of resonant cell design for optoacoustic gas spectroscopy (H-type). Applied Optics, 17, pp. 1110-1119, 1978.

[4] Wolff, M., Groninga, H.G., Baumann, B., Kost, B. \& Harde, H., Resonance investigations using PAS and FEM. Acta Acustica, 91, pp. 1477-1481, Suppl. 1, 99, 2005.

[5] Baumann, B., Kost, B., Groninga, H. \& Wolff, M., Eigenmode analysis of photoacoustic sensors via finite element method. Review of Scientific Instruments, 77, 044901, 2006.

[6] Kost, B., Baumann, B., Wolff, M. \& Groninga, H., Numerical investigations of photoacoustic sensor signals. Acta Acustica united with Acustica, accepted for publication, 2009.

[7] Baumann, B., Wolff, M., Kost, B. \& Groninga, H., Finite element calculation of photoacoustic signals. Applied Optics, 46, pp. 1120-1125, 2007.

[8] Baumann, B., Wolff, M., Kost, B. \& Groninga, H., Solving a coupled field problem by eigenmode expansion and finite element method. International Journal of Multiphysics, 1(3), pp. 303-315, 2007.

[9] Kreuzer, L.B., The physics of signal generation and detection. Optoacoustic Spectroscopy and Detection, ed. Y.H. Pao, Academic: London, pp. 1-25, 1977.

[10] COMSOL-Homepage: www.comsol.com.

[11] VDI-Wärmeatlas. 9. Auflage, Springer-Verlag, 2002.

[12] Rothman, L.S. et al., The HITRAN 2004 molecular spectroscopic database. Journal of Quantitative Spectroscopy \& Radiative Transfer, 96, pp. 139-204, 2005.

[13] Miklós, A., Hess, P. \& Bozóki, Z., Application of acoustic resonators in photoacoustic trace gas analysis and metrology. Review of Scientific Instruments, 72(4), pp. 1937-1955, 2001.

[14] MATLAB-Homepage: www.mathworks.com.

[15] Baumann, B., Kost, B., Wolff, M., Groninga, H., Blöß, T. \& Knickrehm, S., Numerical shape optimization of photoacoustic sample cells: First results. Proceedings of the European COMSOL Conference 2007, Grenoble, France, pp. 1-6, 2007. 